\title{
Constitutive $\beta$-catenin activation in osteoblasts impairs terminal osteoblast differentiation and bone quality
}

Quanwei Bao ${ }^{1}$, Sixu Chen ${ }^{1}$, Hao Qin ${ }^{1}$, Jianquan Feng ${ }^{2}$, Huayu Liu ${ }^{1}$, Daocheng Liu $^{1}$, Ang Li $^{1}$, Yue Shen ${ }^{1}$, Xiaozheng Zhong ${ }^{1}$, Junfeng $\mathrm{Li}^{1}$, and Zhaowen Zong ${ }^{1 *}$

${ }^{1}$ State Key Laboratory of Trauma, Burn and Combined injury, Department of Trauma Surgery, Daping Hospital, Third Military Medical University, ChongQing, 400042, China; ${ }^{2}$ Department of Biomedical Sciences, Baylor College of Dentistry, Texas A\&M Health Science Center, Dallas, TX 75246, USA.

Institutional addresses: State Key Laboratory of Trauma, Burn and Combined Injury, Department of Trauma Surgery, Daping Hospital, Third Military Medical University, Chongqing, 400042, China

* Corresponding author: Zhaowen Zong, State Key Laboratory of Trauma, Burn and Combined injury, Department of Trauma Surgery, Daping Hospital, Third Military Medical University, ChongQing, 400042, China, Cell phone: +86 138-8350-6677; E-mail address: zongzhaowen@sina.cn 


\title{
Constitutive $\beta$-catenin activation in osteoblasts impairs terminal osteoblast differentiation
}

\author{
and bone quality
}

\section{Abstract}

Accumulating evidence suggests that Wnt/ $\beta$-catenin signaling plays a central role in controlling bone mass. We previously reported that constitutive activation of $\beta$-catenin (CA- $\beta$-catenin) in osteoblasts potentially has side effects on the bone growth and bone remodeling process, although it could increase bone mass. The present study aimed to observe the effects of osteoblastic CA- $\beta$-catenin on bone quality and to investigate possible mechanisms of these effects. It was found that CA- $\beta$-catenin mice exhibited lower mineralization levels and disorganized collagen in long bones as confirmed by von Kossa staining and sirius red staining, respectively. Also, bone strength decreased significantly in CA- $\beta$-catenin mice. Then the effect of CA- $\beta$-catenin on biological functions of osteoblasts were investigated and it was found that the expression levels of osteocalcin, a marker for the late differentiation of osteoblasts, decreased in CA- $\beta$-catenin mice, while the expression levels of osterix and alkaline phosphatase, two markers for the early differentiation of osteoblasts, increased in CA- $\beta$-catenin mice. Furthermore, higher proliferation rate were revealed in osteoblasts that were isolated from CA- $\beta$-catenin mice. The Real-time PCR and western blot examination found that the expression level of c-myc and cyclin D1, two G1 progression-related molecules, increased in osteoblasts that were isolated from the CA- $\beta$-catenin mice, and the expression levels of CDK14 and cyclin Y, two mitotic-related molecules that can accelerate cells entering into S and G2/M phases, increased in osteoblasts that were isolated from the CA- $\beta$-catenin mice. In summary, osteoblastic CA- $\beta$-catenin kept osteoblasts in high 
proliferative state and impaired the terminal osteoblast differentiation, and this led to changed bone structure and decreased bone strength.

Key Words: Wnt/ $\beta$-catenin; constitutive activation of $\beta$-catenin; osteoblasts; terminal differentiation; bone quanlity

\section{Introduction}

The evolutionarily conserved Wnt signaling pathway consists of the canonical Wnt/ $\beta$-catenin and non-canonical pathway; these pathways play important roles in many physiological and pathological processes, such as tumorigenesis and the biological functions of various types of stem cells[1-4]. Accumulating evidence suggests that $\mathrm{Wnt} / \beta$-catenin signaling plays a central role in

controlling bone mass. $\beta$-catenin inactivation in mesenchymal progenitors, osteoblasts and osteocytes result in low bone mass, whereas $\beta$-catenin stabilization in mesenchymal progenitors and osteoblasts increases bone mass[3, 5-8]. In addition, inhibition of secreted Wnt antagonists, including sclerostin and dickkopf 1 (DKK1), and intracellular negative regulators of the pathway such as Gsk3ß, could also promote bone formation and increase bone mass in rats or mice[9-11]. These studies revealed that the activation of $\mathrm{Wnt} / \beta$-catenin signaling is a promising approach for the treatment of low bone volume conditions[11, 12].

However, some studies reported that the level of $\mathrm{Wnt} / \beta$-catenin signaling should be precisely controlled to ensure its normal function[13, 14]. We previously reported that bone mass increased significantly after $\beta$-catenin was activated in osteoblasts. However, most of the cancellous bone 
was positive for safranin $\mathrm{O}$ staining when $\beta$-catenin was stabilized at a younger age, indicating that the excessively formed bone was immature[15]. Later, bone strength was found to significantly decrease despite the dramatic increase in cancellous bone mass when $\beta$-catenin was stabilized in osteocytes[16]. These data indicated that constitutive $\beta$-catenin activation in either osteoblasts or osteocytes potentially exhibits sides effects on bone quality, although it could increase bone mass. The underlying mechanism is currently unknown.

More recently, an increasing number of studies showed that the function of Wnt $/ \beta$-catenin signaling is stage dependent and should be finely tuned to ensure its precise control of bone mass and bone remodeling[17-20]. In early osteochondral progenitor cells, a high expression level of Wnt/ $\beta$-catenin signaling promoted osteoblast differentiation and enhanced their growth; however, high Wnt/ß-catenin signaling level in osteoblast lineages would block their differentiation into mature osteoblasts[18]. In our previous study, the immature excessive bone in CA- $\beta$-catenin mice indicated that the late-stage differentiation of osteoblasts is potentially hindered[15]. Therefore, we hypothesized that constitutive activation of $\beta$-catenin in osteoblasts might impair the terminal differentiation of osteoblasts. In the present study, we tested this hypoyesis by observing the effect of osteoblastic CA- $\beta$-catenin on bone quality (i.e. bone structure and bone strength) and investigating the possible mechanisms by observing the effect of osteoblastic CA- $\beta$-catenin on the biological functions of osteoblasts.

\section{Materials and Methods}

\subsection{Animals}

All animal procedures were approved by the Institutional Animal Care and Use Committee of Daping Hospital, Third Military Medical University, P. R. China. All methods were carried out in 
accordance with approved guidelines of the Institutional. Mice expressing the TM-inducible Cre fusion protein, Cre-ER ${ }^{\mathrm{TM}}$ under the control of the $3.2-\mathrm{kb}$ mouse pro-collagen 1 promoter $(3.2 \mathrm{~kb}$ Col1-Cre $\mathrm{ER}^{\mathrm{TM}}$ ) were crossed with Catnb+/lox (exon 3) mice. Genotyping was performed as previously reported [15]. The 3.2-kb Col1-Cre $\mathrm{ER}^{\mathrm{TM}} ; \beta$-catenin exon $3 \mathrm{fx}+/+$ was the target mice in which $\beta$-catenin was constitutively activated in osteoblasts. In contrast, the $\beta$-catenin exon 3 $\mathrm{fx}+/+$ mice, which lacked the 3.2-kb Col1-Cre $\mathrm{ER}^{\mathrm{TM}}$, was used as control. Tamoxifen (TM; Sigma-Aldrich, St. Louis, MO, USA) was injected to activate the function of the promoter. Briefly, TM was dissolved in a small volume of ethanol and diluted with corn oil at a concentration of 10 $\mathrm{mg} / \mathrm{ml}$ [15]. CA- $\beta$-catenin and control mice ( $\mathrm{n}=6$ at each time point) were intraperitoneally injected with TM $(75 \mathrm{mg} / \mathrm{kg})$ twice a week for one month, starting from the age of 3 days postnatal. Because the mice were sexual immature when they were sacrificed (one month of age) and there were no significant difference in the phenotype between male and female mice [16], mice of either sex were used in the following experiments.

\subsection{Radiographic imaging and tissue preparation}

Four weeks after birth, 5'-bromo-2-deoxyuridine (BrdU, Sigma, Ireland) was administered at a dose of $10 \mu 1 /$ g body weight by intraperitoneal injection. After 24 hours, BrdU was injected again at the same dose. Two hours after the injection, the mice were sacrificed by an overdose of anesthetic, and radiographic images of the tibia and femora were obtained using a Faxitron MX-20 system (Faxitron, Wheeling, Illinois). The tibiae and femora were then removed and fixed overnight. Some of the samples were embedded in plastic, and 8- $\mu$ m undecalcified sections were cut for von Kossa staining. The remaining samples were decalcified in $10 \%$ ethylenediaminetetraacetic acid. Once adequately decalcified, the samples were tissue-processed, 
embedded in paraffin, and sectioned at a thickness of $4 \mu \mathrm{m}$ coronally. The sections were de-paraffinized and rehydrated and then used for Sirius Red staining, immunohistochemistry (IHC).

\subsection{Immunohistochemical staining and TdT-mediated dUTP nick end labeling (TUNEL)}

assays

IHC was performed as previously described[15, 16]. The utilized primary antibodies were rabbit anti-goat, osterix (Osx, 1:300), mouse anti-rat alkaline phosphatase (ALP, 1:200), osteocalcin (OCN, 1:300), and BrdU (1:300). The antibodies were purchased from Santa Cruz Biotechnology, Inc., USA. The biotinylated goat anti-mouse, rabbit anti-goat, and goat anti-rabbit IgGs were acquired from Boster (Wuhan, China). To obtain the percentage of cells expressing a given marker protein, photomicrographic images of each section were captured with an Olympus microscope and digital camera under 400x magnification. The number of specific antigen-positive cells was counted in five random fields. The mean and standard deviation of the number of positive cells were calculated for each group and were used for the statistical analysis.

TUNEL was performed according to the manufacturer's instructions (Roche, Basel, Switzerland) and quantified using the same method as described for immunohistochemistry.

\subsection{Sirius Red staining for collagen}

Prepared sections were stained with Weigert's hematoxylin for 8 minutes and then washed for 10 min in running tap water. Then, the slides were stained with picro-Sirius Red for one hour followed by washing in acidified water. The water was removed. The slides were then dehydrated and rinsed in 95\% alcohol for $2 \mathrm{~min}, 100 \%$ alcohol for 3 min (twice), xylene for 2 min (twice), and then cover-slipped with Permount. 


\section{5. von Kossa staining}

von Kossa staining was employed to monitor the mineralization ability of bone. Prepared sections were stained with Silver Nitrate solution, then expose the slides to strong light till mineralized bone turns black. This usually takes 30min-1h. Then put it into Hazardous Waste container followed by aspirating the Silver Nitrate from the slides then rinse with distilled water. Quick dip into 5\% Sodium Thiosulfate $(10 \mathrm{Sec})$. Then Van Gieson Working Solution 5min.Collect the solution to the Waste Base \& Acid Container, don't pour into the sink. The slides were then dehydrated and rinsed in $95 \%$ alcohol for $2 \mathrm{~min}, 100 \%$ alcohol for 3 min (twice), xylene for 2 min (twice), and then cover-slipped with Permount.

\subsection{Mechanical Testing}

Tibia were used for mechanical strength in a four-point bending test using a BOSE ElectroForce ELF 3200 computer-controlled testing machine, which has a force resolution of 0.05 N. During the test, each tibia was placed horizontally on two lower supports that were $6.5 \mathrm{~mm}$ apart, with the anterior surface facing upward. The pressing force was applied vertically to the midshaft of the bone. Each bone was compressed at a speed of $0.05 \mathrm{~mm} / \mathrm{sec}$ until failure, and force-displacement data were collected every $0.01 \mathrm{~s}$. Based on the data, a force-displacement curve was created, and the ultimate force (UF; N) was defined as the bending force at failure. The stiffness was calculated as the slope of the linear portion of the curve.

\subsection{In vitro study}

2.7.1. Osteoblast isolation Osteoblasts were isolated from the long bones of 4-week-old CA- $\beta$-catenin or control mice, according to previously reported protocols[16]. Briefly, the tibiae and femora were removed. The epiphyses were severed, and the bone marrow was flushed out. 
Then, the diaphyses were sectioned into small pieces that were approximately 1 to $2 \mathrm{~mm}^{3}$. The bone pieces were then incubated with $4 \mathrm{ml}$ of collagenase solution at $37^{\circ} \mathrm{C}$ in a shaking water bath for 2 hours to remove all of the remaining soft tissue and adhered cells and then transferred into medium containing 10\% fetal bovine serum (FBS) to inhibit further collagenase activity. The pieces were then rinsed thrice with medium and transferred into $25-\mathrm{cm}^{2}$ flasks at a density of approximately 20 to 30 fragments per flask. The culture medium was replaced thrice weekly.

2.7.2. Real-time polymerase chain reaction (PCR) RNA was isolated from cultured osteoblasts using TRIzol reagent (Invitrogen) according to the manufacturer's instructions. Then, real-time PCR with the SYBR green detection method was used to examine the $\beta$-catenin, c-myc, cyclin Y, cyclin D1, Cyclin-Dependent Kinase 14(CDK14), OSX, ALP and OCN expression levels. Glyceraldehyde-3-phosphate dehydrogenase (GAPDH) served as a control, and the expression levels of a given gene were expressed as the proportion relative to the mean GAPDH value. The primers that were used are presented in table 1 .

2.7.3. Mineralization induction and Alizarin red staining Alizarin red staining was employed to monitor the mineralization ability of the isolated osteoblasts. Briefly, passaged osteoblasts were cultured for 3 days. Then, osteogenic differentiation medium (DMEM supplemented with $10 \% \mathrm{FBS}, 100 \mathrm{nM}$ dexamethasone, $10 \mathrm{mM} \beta$-glycerophosphate, $250 \mathrm{mM}$ ascorbic acid, $1 \%$ penicillin and $1 \%$ streptomycin) was added to the plates. Then, after 14 days, the cells were stained with $0.005 \%$ Alizarin Red $\mathrm{S}$ in $0.2 \mathrm{~N} \mathrm{KOH}$ for $15 \mathrm{~min}$ to observe the mineralization level.

2.7.4. Western blotting The cultured osteoblasts were harvested by centrifugation, and the cell pellets were collected. The total protein was extracted using cell lysis buffer for western 
(Beyotime) according to the manufacturer's instructions. After the protein concentrations were determined, the samples were separated via $15 \%$ sodium dodecyl sulfate polyacrylamide gel electrophoresis (SDS-PAGE). The protein was then transferred onto polyvinyl difluoride (PVDF) membranes using the Trans-Blot Semi-Dry Transfer Cell (Bio-Rad) and blocked in 5\% skim milk. The membranes were probed with anti- $\beta$-catenin, c-myc, cyclin Y, cyclin D1, CDK14, Bax and Bcl-2 rabbit polyclonal antibodies followed by biotinylated goat anti-rabbit IgG (Boster, Wuhan, China; diluted to a concentration of $10 \mathrm{~g} / \mathrm{ml}$ ). Protein expression was visualized using luminol-enhanced chemiluminescence and were densitometrically assessed. $\beta$-actin served as a control, and the expression levels of a given protein were expressed as the proportion relative to the mean $\beta$-actin value.

2.7.5. Cell proliferation and TUNEL assays BrdU was added to the culture medium at a dose of $10 \mu \mathrm{l} / \mathrm{ml}$. Twenty-four hours later, the cells were fixed with $90 \%$ ethanol and then IHC against BrdU was performed as the above-mentioned method. TUNEL staining was performed and quantified using the same method as described above.

2.7.6. Cell cycle examination The cultured osteoblasts cells were digested with trypsin, carefully collected, washed twice with cold phosphate-buffered saline (PBS), and then fixed in $70 \%$ ethanol overnight at $-20^{\circ} \mathrm{C}$. On the following day, the ethanol was removed. The samples were washed thrice with PBS and then stained with PI/RNase $(50 \mu \mathrm{g} / \mathrm{ml} / 100 \mu \mathrm{l} / \mathrm{ml})$ at room temperature for $30 \mathrm{~min}$. The samples were analyzed with a FC 500 MCL Flow Cytometer (Beckman Coulter).

\subsection{Statistical analysis}

All of the data were expressed as means \pm standard deviation. Statistical significance was 
evaluated with one-way analysis of variance (ANOVA) using the SPSS 11.0 software. Data were considered significant at $P<0.05$.

\section{Results}

\subsection{Osteoblastic CA-ß-catenin changed bone structure and lowered bone strength}

After $\beta$-catenin was stabilized in osteoblasts, massive bone mass formed in bone marrow cavity of long bones as previously reported (data not shown)[15]. However, Sirius Red staining demonstrated that the arrangement of collagen in both trabecular and cortical bone of the CA- $\beta$-catenin mice were more disorganized when compared with control mice (Fig. 1A, B). Regarding mineralization, we observed that the mineralization level in the CA- $\beta$-catenin mice was reduced compared with that in control mice (Fig. 1C). The mineralization ability of the osteoblasts that were isolated from the CA- $\beta$-catenin mice was also reduced compared with control mice (Fig. 1D). Taken together, these data indicated that collagen re-arrangement and the mineralization process were impaired in the CA- $\beta$-catenin mice.

Mechanically, it was revealed that bone strength decreased significantly in CA- $\beta$-catenin mice, as shown by decreases in the ultimate load and stiffness (Fig. 2A). Also, spontaneous fractures of the long bone were constantly found in CA- $\beta$-catenin mice as confirmed by X-ray radiography (Fig. 2B).

\subsection{Osteoblastic CA-p-catenin kept osteoblasts in high proliferative state and impaired the}

\section{terminal osteoblast differentiation}

Cell proliferation and death synergistically control the cell number. Compared with control mice, the number of BrdU-positive osteoblasts in the CA- $\beta$-catenin mice was increased, whereas the TUNNEL-positive osteoblasts in the CA- $\beta$-catenin mice were reduced (Fig. 3A-D). These 
findings were further confirmed by in vitro studies (Fig. 3E-H). The Bax is a water soluble protein which is homologous with BCL-2, and it is a cell apoptosis promoting gene in BCL-2 gene family. The over expression of Bax can inhibit the protective effect of BCL-2 and cause the cell to die. The study found that the ratio between $\mathrm{Bax} / \mathrm{Bcl}-2$ two protein is the key factor to determine the inhibition of apoptosis, and therefore, Bax is one of the most important genes to promote cell death (Apoptosis). Western blot analysis revealed that the expression levels of Bcl-2 increased in osteoblasts that were isolated from the CA- $\beta$-catenin mice, and the expression levels of Bax decreased in osteoblasts that were isolated from the CA- $\beta$-catenin mice, whereas the ratio of Bax to Bcl-2 decreased in osteoblasts that were isolated from the CA- $\beta$-catenin mice(Fig. 3I-K). Taken together, these findings indicated that osteoblasts were kept in a highly proliferative state in the CA- $\beta$-catenin mice.

We then investigated the mechanisms for the highly proliferative state of osteoblasts in the CA- $\beta$-catenin mice. Cell cycle analysis revealed that more osteoblasts that were isolated from the CA- $\beta$-catenin mice were captured in the $S$ and $\mathrm{G} 2 / \mathrm{M}$ stages when compared with the control mice((Fig. 4A, B). Western blot analysis revealed that the expression levels of c-myc and cyclin D1 (two G1 progression-related genes), CDK14 and cyclin Y (two mitotic-related genes that can accelerate cells entering into $\mathrm{S}$ and $\mathrm{G} 2 / \mathrm{M}$ phases) increased in osteoblasts that were isolated from the CA- $\beta$-catenin mice (Fig. 4C, D). Real-time PCR examination also confirmed the findings of the western blot analysis (Fig. 4E).

Osterix and alkaline phosphatase are two markers for the early differentiation of osteoblasts[21-24]. We found that the expression level of osterix and alkaline phosphatase increased in long bones of CA- $\beta$-catenin mice (Fig. 5A-D). Additionally, immunohistochemistry 
examination revealed that the osterix expression was not only observed in osteoblasts but also in osteocytes, indicating that these osteocytes were not fully differentiated [25](Fig.5A, arrowheads). In contrast, the expression level of osteocalcin, a marker for the terminal differentiation of osteoblasts[21, 22, 24] decreased in CA- $\beta$-catenin mice when compared with control mice (Fig. 5E-F). These findings were further confirmed by real-time PCR (Fig. 5G).

Taken together, these data indicated that osteoblasts were maintained in a highly proliferative state in the CA- $\beta$-catenin mice and that increased expression of G1 progression-related genes (c-myc and cyclin D1) and mitotic-related genes (CDK14 and cyclin Y) contributed to this change; and terminal osteoblasts differentiation was impaired in the CA- $\beta$-catenin mice.

\section{Discussion}

Bone mass is adjusted by two main processes, namely, bone formation and bone resorption; these processes involve three main types of cells in bone: osteoblasts, osteoclasts and osteocytes. Osteoblasts play an important role in controlling bone mass because they are the main cells responsible for bone formation; they also play a pivotal role in regulating osteoclast genesis and function[6-8]. During the early stage of bone formation, osteoblasts secrete osteoid, forming the scaffolding for new bone. Then, osteoblasts secrete noncollagenous organic proteins to induce the terminal differentiation of osteoblasts and mineralization of the osteoid[21-23, 26]. The function of osteoblasts is thought to be regulated by various hormones and by locally acting growth factors, among which the Wnt/ $\beta$-catenin signaling pathway has been shown to be one of the most important. Accumulating evidence suggests that activation of $\mathrm{Wnt} / \beta$-catenin in osteoblasts by various means results in increased bone mass and favors osteoblast differentiation [6-8, 27]. However, more recent studies have shown that the function of $\mathrm{Wnt} / \beta$-catenin signaling is stage 
dependent, that is, Wnt/ $\beta$-catenin signaling can promote the genesis of osteoblasts from mesenchymal stem cells and the early differentiation of osteoblasts but can repress the mineralization process of osteoblasts[13, 17-20, 24, 28]. Thus, Wnt/ $\beta$-catenin signaling should be finely tuned; otherwise, this signaling may lead to various adverse effects[14-16]. After $\beta$-catenin was constitutively activated in osteoblasts in the current study, the early differentiation of osteoblasts was not disturbed and the expression levels of early differentiation markers, such as alkaline phosphatase and osterix, were up-regulated. In contrast, osteoblasts terminal differentiation was impaired, as indicated by the decreased mineralization level, disorganized collagen arrangement and down-regulated osteocalcin expression.

Bone quality is controlled by the intrinsic properties of bone material and structure. Material properties primarily refer to the degree of mineralization and collagen characteristics, and structural properties mainly refer to the diameter and thickness of the cortices and trabecular bone mass[29]. Bone strength can be defined as the ability of bone to resist fracture, which depends on bone mass and on bone quality[29]. As reported previously, trabecular bone mass increased significantly in CA- $\beta$-catenin mice; however, bone strength decreased $[15,16]$. This decrease in bone strength was caused by decreased bone material properties, which were attributed to impaired osteoblast terminal differentiation when $\beta$-catenin was constitutively activated. Thus, Wnt/ $\beta$-catenin signaling should be kept at a proper level to ensure terminal osteoblast differentiation, consequently, ensureing the bone quality and bone strength.

Another finding of the present study is that osteoblasts were maintained in a highly proliferative state when $\beta$-catenin was constitutively activated, and increased expression of G1 progression-related genes (c-myc and cyclin D1) and mitotic-related genes (CDK14 and cyclin Y) 
contributed to these changes. This finding is consistent with previous reports that $\beta$-catenin/Tcf-Lef complex accumulation in the nucleus could promote cell cycle progression and proliferation by up-regulating G1 progression-related genes and mitotic-related genes[30, 31]. Recently, the increased levels of the mitotic CDK14/cyclin Y complex in S and G2 phase cells were shown to up-regulate Wnt- $\beta$-catenin signaling through phosphorylation of the LRP6 co-receptor[32, 33]. Thus, a positive feedback loop formed between the CDK14/cyclin Y complex and $\beta$-catenin activation; in particular, constitutive activation of $\beta$-catenin will up-regulate the expression levels of CDK14 and cyclin Y, while the up-regulated expression of CDK14 and cyclin Y will subsequently enhance the expression of $\beta$-catenin[32, 33]. This positive feedback loop would promote cell cycle progression and maintain cells in a highly proliferative state. This possibility raises two concerns. One concern is that the highly proliferative state of osteoblasts might lead to tumorigenesis because hyper-activation of Wnt signaling in various tissues has been shown to trigger uncontrolled cell proliferation and eventually lead to cancer[30, 34, 35]. Another concern is that if cells are kept in a proliferative state and cannot exit the cell cycle, proliferation-to-differentiation transition will be hindered and cell differentiation will eventually be impaired[28, 36, 37]. During the investigation of the role of osterix in controlling cementoblast differentiation, Cao et al. found that $\mathrm{Wnt} / \beta$-catenin signaling must be kept a low level to ensure the differentiation of cementoblasts. These authors proposed that a high level of Wnt/ $\beta$-catenin signaling would lead to a greater mass of immature cementum due to an increase in cell proliferation coupled with a decrease in cell differentiation[28]. Considering the high similarity between cementoblasts and osteoblasts, we proposed that the highly proliferative state of osteoblasts in CA- $\beta$-catenin mice might be one possible mechanism for the impaired terminal 
differentiation of osteoblasts. While Liu etc. found that the cell proliferation was enhanced, cell differentiation and mineralization were inhibited when RANKL/OPG was significantly decreased[38]. In our study the RANKL/OPG was significantly decreased in CA- $\beta$-catenin mice (data not shown), so some of the osteoblastic phenotypes observed in vivo here (increased proliferation and reduced differentiation) may be secondary to defective osteoclast activity. However, these two possible mechanistic speculations require testing by further studies.

In summary, the present study revealed that the constitutive activation of $\beta$-catenin in osteoblasts could increase cancellous bone mass; however, the activation also had adverse effects on bone structure and strength. The osteoblasts of CA- $\beta$-catenin mice kept in high proliferative state and impaired the terminal osteoblast differentiation might be the possible mechanism. These adverse effects should be addressed before the adoption of any therapeutic clinical application involving adjustment of the $\mathrm{Wnt} / \beta$-catenin signaling pathway.

\section{Competing interests}

All authors report no conflicts of interest.

\section{Acknowledgements}

This work was funded by Foundation of State Key Laboratory of Trauma, Burns and combined injury (SKLZZ201124), and National Science Foundation of China (81271935). 


\section{References}

[1] C. Ma, C. Zeng, L. Jin, Y. Yang, P. Li, L. Chen, J. Wang, GSK3beta mediates the carcinogenic effect of HPV16 in cervical cancer, Scientific reports 5 (2015) 16555.

[2] J. Velasco, M.T. Zarrabeitia, J.R. Prieto, J.L. Perez-Castrillon, M.D. Perez-Aguilar, M.I. Perez-Nunez, C. Sanudo, J. Hernandez-Elena, I. Calvo, F. Ortiz, J. Gonzalez-Macias, J.A. Riancho, Wnt pathway genes in osteoporosis and osteoarthritis: differential expression and genetic association study, Osteoporosis international : a journal established as result of cooperation between the European Foundation for Osteoporosis and the National Osteoporosis Foundation of the USA 21 (2010) 109-118.

[3] Y. Wu, F. Liu, Y. Liu, X. Liu, Z. Ai, Z. Guo, Y. Zhang, GSK3 inhibitors CHIR99021 and 6-bromoindirubin-3'-oxime inhibit microRNA maturation in mouse embryonic stem cells, Scientific reports 5 (2015) 8666.

[4] J. Wang, Spatial orientation of the microscopic elements of cortical repair bone, Clinical orthopaedics and related research (2000) 265-277.

[5] J. Chen, F. Long, beta-catenin promotes bone formation and suppresses bone resorption in postnatal growing mice, Journal of bone and mineral research : the official journal of the American Society for Bone and Mineral Research 28 (2013) 1160-1169.

[6] C. Zuo, Y. Huang, R. Bajis, M. Sahih, Y.P. Li, K. Dai, X. Zhang, Osteoblastogenesis regulation signals in bone remodeling, Osteoporosis international : a journal established as result of cooperation between the European Foundation for Osteoporosis and the National Osteoporosis Foundation of the USA 23 (2012) 1653-1663.

[7] S.L. Holmen, C.R. Zylstra, A. Mukherjee, R.E. Sigler, M.C. Faugere, M.L. Bouxsein, L. Deng, 
T.L. Clemens, B.O. Williams, Essential role of beta-catenin in postnatal bone acquisition, The Journal of biological chemistry 280 (2005) 21162-21168.

[8] D.A. Glass, 2nd, P. Bialek, J.D. Ahn, M. Starbuck, M.S. Patel, H. Clevers, M.M. Taketo, F. Long, A.P. McMahon, R.A. Lang, G. Karsenty, Canonical Wnt signaling in differentiated osteoblasts controls osteoclast differentiation, Developmental cell 8 (2005) 751-764.

[9] J. Guo, M. Liu, D. Yang, M.L. Bouxsein, H. Saito, R.J. Galvin, S.A. Kuhstoss, C.C. Thomas, E. Schipani, R. Baron, F.R. Bringhurst, H.M. Kronenberg, Suppression of Wnt signaling by Dkk1 attenuates PTH-mediated stromal cell response and new bone formation, Cell metabolism 11 (2010) 161-171.

[10] P. Clement-Lacroix, M. Ai, F. Morvan, S. Roman-Roman, B. Vayssiere, C. Belleville, K. Estrera, M.L. Warman, R. Baron, G. Rawadi, Lrp5-independent activation of Wnt signaling by lithium chloride increases bone formation and bone mass in mice, Proceedings of the National Academy of Sciences of the United States of America 102 (2005) 17406-17411.

[11] D. Padhi, G. Jang, B. Stouch, L. Fang, E. Posvar, Single-dose, placebo-controlled, randomized study of AMG 785, a sclerostin monoclonal antibody, Journal of bone and mineral research : the official journal of the American Society for Bone and Mineral Research 26 (2011) 19-26.

[12] R. Sapir-Koren, G. Livshits, Osteocyte control of bone remodeling: is sclerostin a key molecular coordinator of the balanced bone resorption-formation cycles?, Osteoporosis international : a journal established as result of cooperation between the European Foundation for Osteoporosis and the National Osteoporosis Foundation of the USA 25 (2014) 2685-2700.

[13] M. Eijken, I.M. Meijer, I. Westbroek, M. Koedam, H. Chiba, A.G. Uitterlinden, H.A. Pols, J.P. van Leeuwen, Wnt signaling acts and is regulated in a human osteoblast differentiation 
dependent manner, Journal of cellular biochemistry 104 (2008) 568-579.

[14] A. Kode, J.S. Manavalan, I. Mosialou, G. Bhagat, C.V. Rathinam, N. Luo, H. Khiabanian, A. Lee, V.V. Murty, R. Friedman, A. Brum, D. Park, N. Galili, S. Mukherjee, J. Teruya-Feldstein, A. Raza, R. Rabadan, E. Berman, S. Kousteni, Leukaemogenesis induced by an activating beta-catenin mutation in osteoblasts, Nature 506 (2014) 240-244.

[15] M. Jia, S. Chen, B. Zhang, H. Liang, J. Feng, Z. Zong, Effects of constitutive beta-catenin activation on vertebral bone growth and remodeling at different postnatal stages in mice, PloS one 8 (2013) e74093.

[16] S. Chen, J. Feng, Q. Bao, A. Li, B. Zhang, Y. Shen, Y. Zhao, Q. Guo, J. Jing, S. Lin, Z. Zong, Adverse Effects of Osteocytic Constitutive Activation of ss-Catenin on Bone Strength and Bone Growth, Journal of bone and mineral research : the official journal of the American Society for Bone and Mineral Research 30 (2015) 1184-1194.

[17] T.F. Day, Y. Yang, Wnt and hedgehog signaling pathways in bone development, The Journal of bone and joint surgery. American volume 90 Suppl 1 (2008) 19-24.

[18] J.B. Regard, N. Cherman, D. Palmer, S.A. Kuznetsov, F.S. Celi, J.M. Guettier, M. Chen, N. Bhattacharyya, J. Wess, S.R. Coughlin, L.S. Weinstein, M.T. Collins, P.G. Robey, Y. Yang, Wnt/beta-catenin signaling is differentially regulated by Galpha proteins and contributes to fibrous dysplasia, Proceedings of the National Academy of Sciences of the United States of America 108 (2011) 20101-20106.

[19] Y. Chen, H.C. Whetstone, A.C. Lin, P. Nadesan, Q. Wei, R. Poon, B.A. Alman, Beta-catenin signaling plays a disparate role in different phases of fracture repair: implications for therapy to improve bone healing, PLoS medicine 4 (2007) e249. 
[20] G. van der Horst, S.M. van der Werf, H. Farih-Sips, R.L. van Bezooijen, C.W. Lowik, M. Karperien, Downregulation of Wnt signaling by increased expression of Dickkopf-1 and -2 is a prerequisite for late-stage osteoblast differentiation of KS483 cells, Journal of bone and mineral research : the official journal of the American Society for Bone and Mineral Research 20 (2005) 1867-1877.

[21] Y. Nishikawa, Y. Akiyama, K. Yamamoto, M. Kobayashi, E. Watanabe, N. Watanabe, N. Shimizu, Y. Mikami, K. Komiyama, Osteocytes up-regulate the terminal differentiation of pre-osteoblasts via gap junctions, Biochemical and biophysical research communications 456 (2015) 1-6.

[22] K. Uchihashi, S. Aoki, A. Matsunobu, S. Toda, Osteoblast migration into type I collagen gel and differentiation to osteocyte-like cells within a self-produced mineralized matrix: a novel system for analyzing differentiation from osteoblast to osteocyte, Bone 52 (2013) 102-110.

[23] F. Liu, F. Fang, H. Yuan, D. Yang, Y. Chen, L. Williams, S.A. Goldstein, P.H. Krebsbach, J.L. Guan, Suppression of autophagy by FIP200 deletion leads to osteopenia in mice through the inhibition of osteoblast terminal differentiation, Journal of bone and mineral research : the official journal of the American Society for Bone and Mineral Research 28 (2013) 2414-2430.

[24] C. Martineau, O. Kevorkova, L. Brissette, R. Moreau, Scavenger receptor class B, type I (Scarb1) deficiency promotes osteoblastogenesis but stunts terminal osteocyte differentiation, Physiological reports 2 (2014).

[25] J.Q. Feng, L.M. Ward, S. Liu, Y. Lu, Y. Xie, B. Yuan, X. Yu, F. Rauch, S.I. Davis, S. Zhang, H. Rios, M.K. Drezner, L.D. Quarles, L.F. Bonewald, K.E. White, Loss of DMP1 causes rickets and osteomalacia and identifies a role for osteocytes in mineral metabolism, Nature genetics 
38 (2006) 1310-1315.

[26] K. Kawasaki, A.V. Buchanan, K.M. Weiss, Biomineralization in humans: making the hard choices in life, Annual review of genetics 43 (2009) 119-142.

[27] J.H. Hwang, P.H. Cha, G. Han, T.T. Bach, S. Min do, K.Y. Choi, Euodia sutchuenensis Dode extract stimulates osteoblast differentiation via Wnt/beta-catenin pathway activation, Experimental \& molecular medicine 47 (2015) e152.

[28] Z. Cao, R. Liu, H. Zhang, H. Liao, Y. Zhang, R.J. Hinton, J.Q. Feng, Osterix controls cementoblast differentiation through downregulation of Wnt-signaling via enhancing DKK1 expression, International journal of biological sciences 11 (2015) 335-344.

[29] K.S. Davison, K. Siminoski, J.D. Adachi, D.A. Hanley, D. Goltzman, A.B. Hodsman, R. Josse, S. Kaiser, W.P. Olszynski, A. Papaioannou, L.G. Ste-Marie, D.L. Kendler, A. Tenenhouse, J.P. Brown, Bone strength: the whole is greater than the sum of its parts, Seminars in arthritis and rheumatism 36 (2006) 22-31.

[30] O. Tetsu, F. McCormick, Beta-catenin regulates expression of cyclin D1 in colon carcinoma cells, Nature 398 (1999) 422-426.

[31] T.C. He, A.B. Sparks, C. Rago, H. Hermeking, L. Zawel, L.T. da Costa, P.J. Morin, B. Vogelstein, K.W. Kinzler, Identification of c-MYC as a target of the APC pathway, Science 281 (1998) 1509-1512.

[32] Y. Ding, S. Su, W. Tang, X. Zhang, S. Chen, G. Zhu, J. Liang, W. Wei, Y. Guo, L. Liu, Y.G. Chen, W. Wu, Enrichment of the beta-catenin-TCF complex at the S and G2 phases ensures cell survival and cell cycle progression, Journal of cell science 127 (2014) 4833-4845.

[33] G. Davidson, J. Shen, Y.L. Huang, Y. Su, E. Karaulanov, K. Bartscherer, C. Hassler, P. Stannek, 
M. Boutros, C. Niehrs, Cell cycle control of wnt receptor activation, Developmental cell 17 (2009) 788-799.

[34] C. Chen, M. Zhao, A. Tian, X. Zhang, Z. Yao, X. Ma, Aberrant activation of Wnt/beta-catenin signaling drives proliferation of bone sarcoma cells, Oncotarget 6 (2015) 17570-17583.

[35] H. Clevers, R. Nusse, Wnt/beta-catenin signaling and disease, Cell 149 (2012) 1192-1205.

[36] G.M. Boland, G. Perkins, D.J. Hall, R.S. Tuan, Wnt 3a promotes proliferation and suppresses osteogenic differentiation of adult human mesenchymal stem cells, Journal of cellular biochemistry 93 (2004) 1210-1230.

[37] S. Minear, P. Leucht, J. Jiang, B. Liu, A. Zeng, C. Fuerer, R. Nusse, J.A. Helms, Wnt proteins promote bone regeneration, Science translational medicine 2 (2010) 29ra30.

[38] Hai-Juan Liu, Hua Yan, Jun Yan, Hao Li, Liang Chen, Li-Ren Han, and Xiao-Fei Yang, Substance P Promotes the Proliferation, but Inhibits Differentiation and Mineralization of Osteoblasts from Rats with Spinal Cord Injury via RANKL/OPG System, PLoS One. 2016; 11(10): e0165063. 
Table 1. Primers used for real-time PCR

\begin{tabular}{|c|c|}
\hline Genes & Primers \\
\hline \multirow{2}{*}{$\beta$-catenin } & F: 5'-ATGGAGCCGGACAGAAAAGC-3', \\
\hline & R: 5'-CTTGCCACTCAGGGAAGGA-3' \\
\hline \multirow{2}{*}{ c-myc } & F:5'- ATGCCCCTCAACGTGAACTTC-3' \\
\hline & R:5'- CGCAACATAGGATGGAGAGCA-3' \\
\hline \multirow{2}{*}{ cyclinD1 } & F:5'- GCGTACCCTGACACCAATCTC-3' \\
\hline & R:5'- CTCCTCTTCGCACTTCTGCTC-3' \\
\hline \multirow{2}{*}{ CDK 14} & F:5'- GAAAGGACTAAAGCACGCCAA-3' \\
\hline & R:5'- AGACCCCTTCTTATCTCGCTTAC-3' \\
\hline \multirow{2}{*}{ cyclin $\mathrm{Y}$} & F:5'-CAGACGGACGTGAGAGAAAAA-3' \\
\hline & R:5'-TGGTTGACTGACTGTGCTATCA-3' \\
\hline \multirow{2}{*}{ ALP } & F:5'- ACGAGATGCCACCAGAGG-3' \\
\hline & R:5'- AGTTCAGTGCGGTTCCAG -3' \\
\hline \multirow{2}{*}{ OSX } & F:5-CTCCTGCGACTGCCCTAA-3 \\
\hline & R:5-ACATACCGTTCCGAAGCG-3 \\
\hline \multirow{2}{*}{ OCN } & F:5-AGGGCAGCGAGGTAGTGA-3 \\
\hline & R:5-TGGTGTAGCCGAAAGTCC-3 \\
\hline \multirow{2}{*}{ GAPDH } & F: 5'-TCACTGCCACCCAGAAGA -3', \\
\hline & R: 5'-AAGTCGCAGGAGACAACC -3'. \\
\hline
\end{tabular}




\section{Figure legends}

Figure 1 - Collagen arrangement and mineralization levels were disturbed in CA- $\beta$-catenin mice. (A-B) Sirius Red staining of tibiae in CA- $\beta$-catenin and control mice. Magnification: 400x ; (C) von Kossa staining of undecalcified tibial in CA- $\beta$-catenin mice and control mice. Magnification: 40x; (D) The mineralization levels of three groups of primary osteoblasts isolated from CA- $\beta$-catenin mice and control mice (arrows: mineralized nodul), Magnification: 40x. All mice cited above were at 4 weeks of age ( $\mathrm{n}=6 /$ group).

Figure 2- Mechanical test and X-ray radiology examination results in each group. (A-B) The ultimate load and stiffness of tibia in CA- $\beta$-catenin mice and control mice; (C-D) Representative X-ray radiographs of long bone in CA- $\beta$-catenin mice and control mice ( arrowhead: spontaneous fractures); All mice cited above were at 4 weeks of age. Values are expressed as the means $\pm \mathrm{SD}$. *Significantly different from control group $(* *: P<0.01 ; \mathrm{n}=6 /$ group $)$.

Figure 3- Constitutive activation of $\beta$-catenin in osteoblastes affected osteoblasts proiferation

and apoptosis. $(\mathrm{A}, \mathrm{B}) \mathrm{IHC}$ staining of $\mathrm{BrdU}$ of tibiae of CA- $\beta$-catenin mice and control mice, the number of Brdu-positive cells (arrows) was increased in CA- $\beta$-catenin mice. Magnification: 400x; (C,D) TUNEL assay on paraffin tibial sections of CA- $\beta$-catenin mice and control mice, the number of apoptotic cells in the cancellous bone of tibiae (arrowsheads) was decresed in CA- $\beta$-catenin mice. Magnification: 400x; (E,F) Fluorescent immunocytochemistry for BrdU in osteoblasts (red arrowheads) isolated from CA- $\beta$-catenin mice and control mice. Magnification: 400x; (G,H) TUNEL assay in osteoblasts isolated from CA- $\beta$-catenin mice, and control mice (yellow arrowheads: apoptotic cells). Magnification: 400x; (I-K) The expression of Bax, Bcl-2 
proteins in primary osteoblasts from CA- $\beta$-catenin mice and control mice was examined by western blot assays. All mice cited above were at 4 weeks of age. Values are expressed as the means \pm SD. *Significantly different from control group $(*: P<0.05 ; * *: P<0.01 ; \mathrm{n}=6 /$ group$)$.

Figure 4- Changes of cell cycle-related molecules in CA- $\beta$-catenin mice. (A) The Cell cycle results detected by flow cytometry analysis of osteoblasts isolated from CA- $\beta$-catenin mice and control mice; (B) Quantitative analysis of measured histograms: \% of cells in S+G2/M phase; (C,D) The expression of $\beta$-catenin, c-myc, cyclin D1, cyclin Y and CDK14 proteins in primary osteoblasts from CA- $\beta$-catenin mice and control mice was examined by western blot assays; (E) The $\beta$-catenin, c-myc, cyclin D1, cyclin Y, CDK14 mRNA expression levels in CA- $\beta$-catenin mice and control mice. All mice cited above were at 4 weeks of age. Values are expressed as the means \pm SD. *Significantly different from control group $(* *: P<0.01 ; \mathrm{n}=6 /$ group$)$.

Figure 5- The exprssion of osteoblast differentiation markers in CA- $\beta$-catenin mice and control mice. (A-D) Anti-Osterix and ALP (the early differentiation markers of osteoblasts) IHC analysis on paraffin tibial sections of CA- $\beta$-catenin mice and control mice revealed that the number of Osterix and ALP-positive osteoblasts (arrows) was increased in CA- $\beta$-catenin mice, and also has some Osterix -positive osteocytes (arrowheads). Magnification: 400x; (E-F) Anti-OCN (the terminal differentiation markers of osteoblasts) IHC analysis on paraffin tibial sections of CA- $\beta$-catenin mice and control mice revealed that the number of OCN positive osteoblasts (arrows) was decreased in CA- $\beta$-catenin mice; (G) The OSX, ALP, OCN mRNA expression levels in CA- $\beta$-catenin mice and control mice. Magnification: 400x. All mice cited 
above were at 4 weeks of age. Values are expressed as the means \pm SD. *Significantly different from control group $(*: P<0.05 ; * *: P<0.01 ; \mathrm{n}=6 /$ group $)$. 


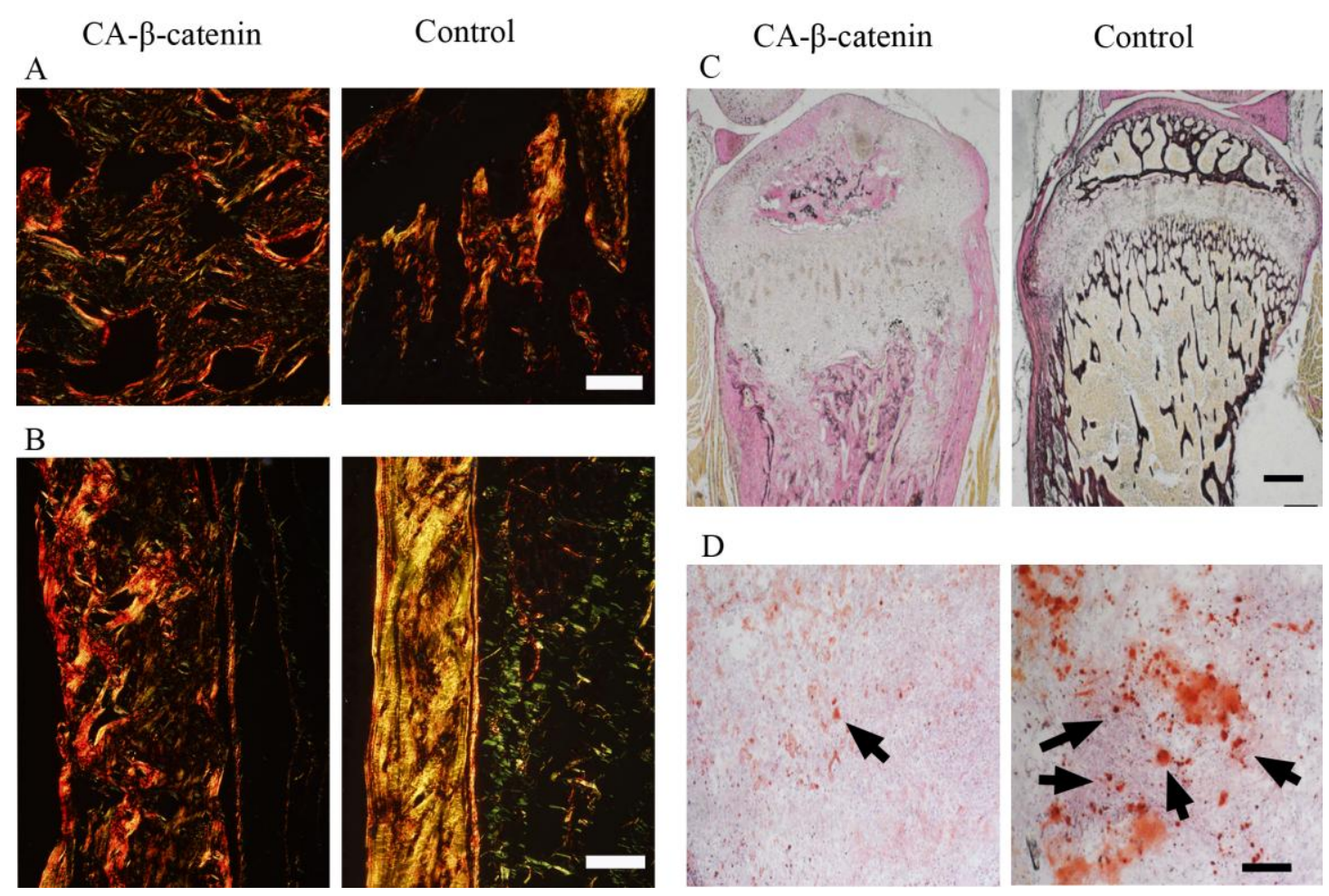




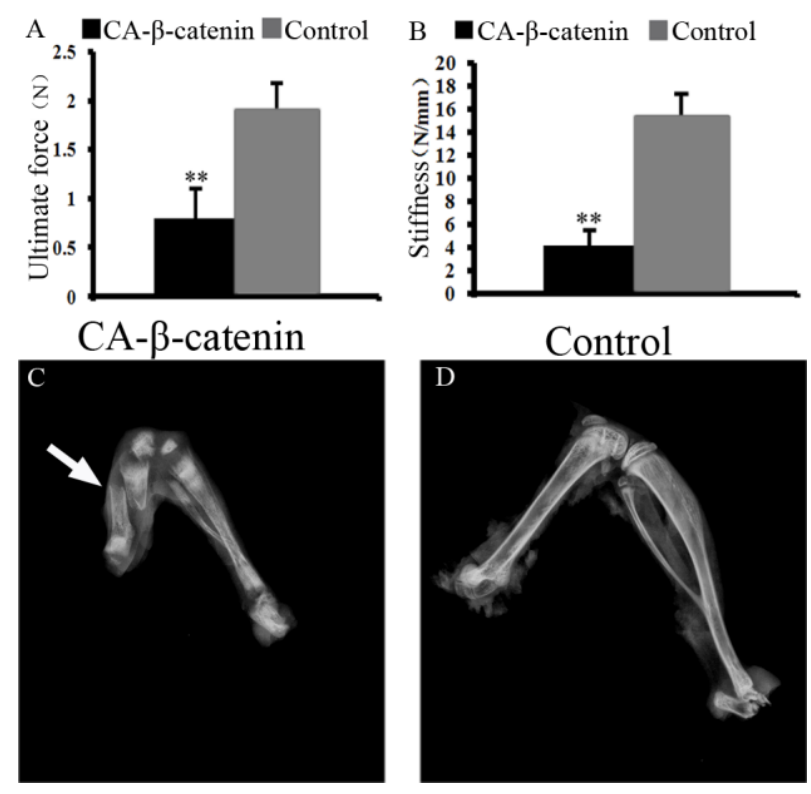


A

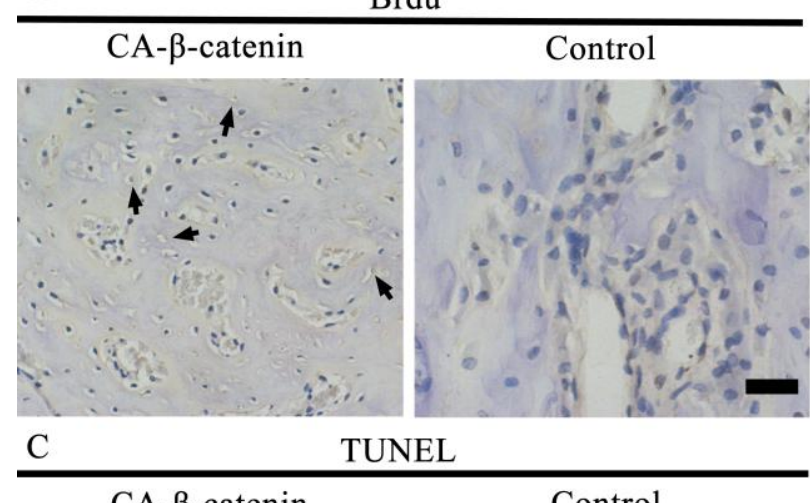

$\frac{\mathrm{C}}{\text { CA- } \beta \text {-catenin }}$
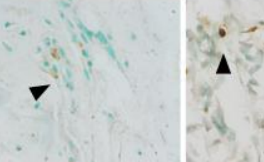

$4 i$
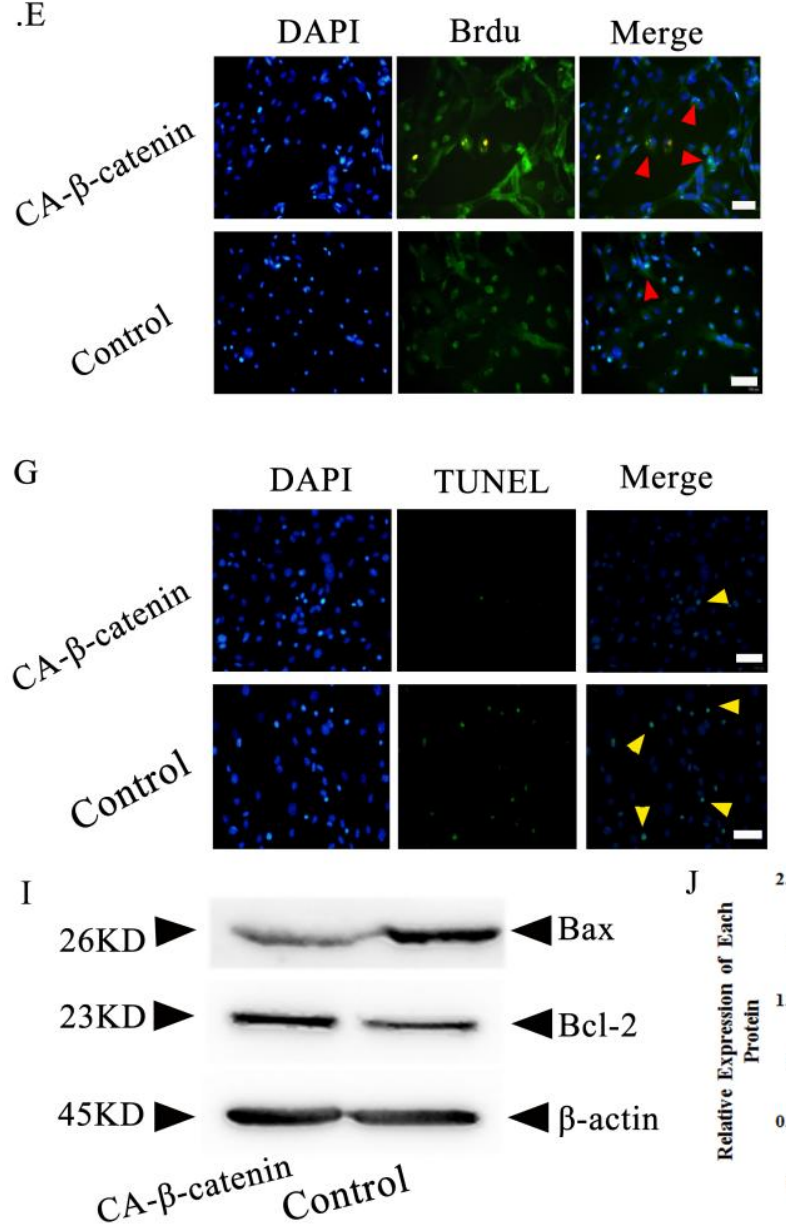

B

-CA- $\beta$-catenin $\quad$ Control

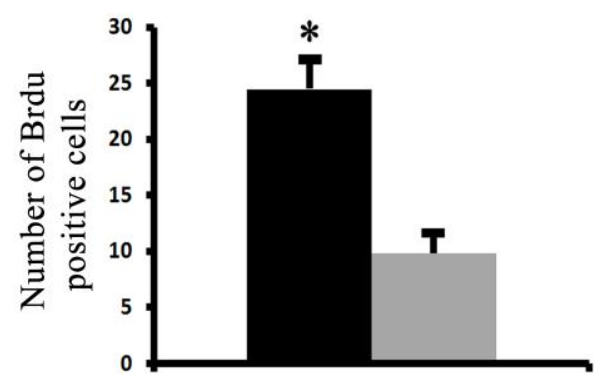

D

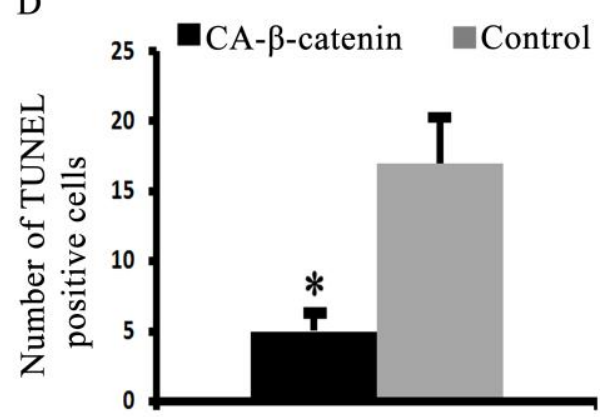

F

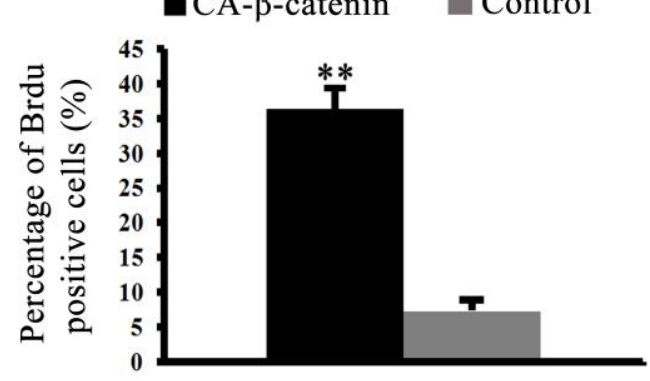

$\mathrm{H}$ - CA- $\beta$-catenin Control

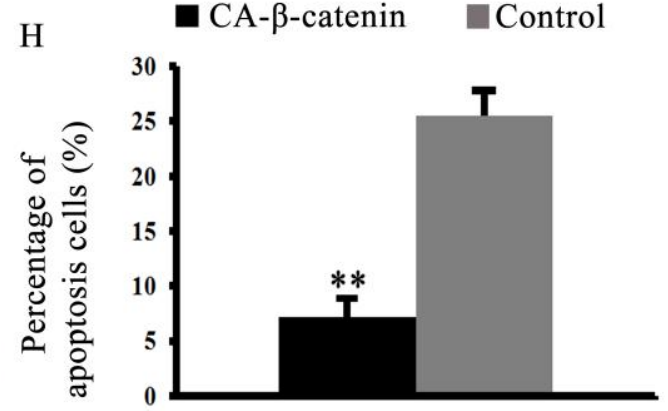

$\mathrm{H}$
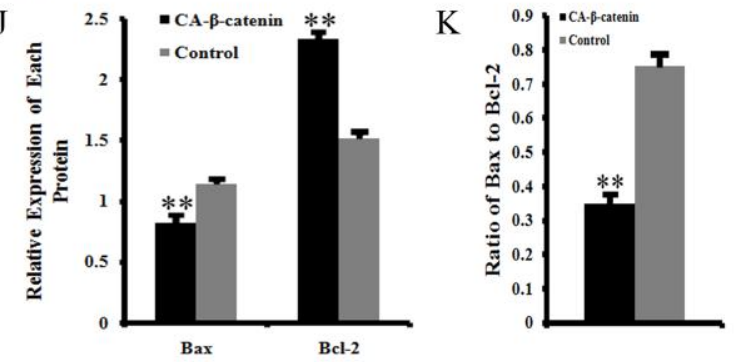

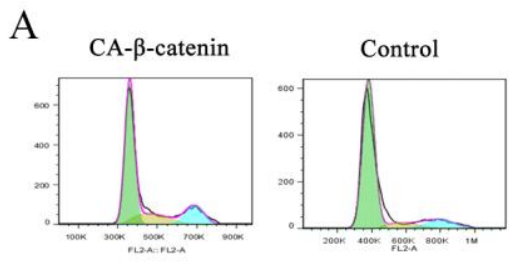

C
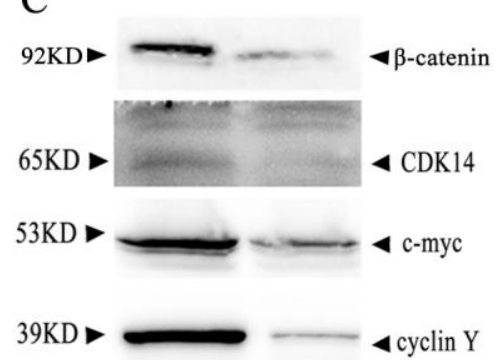

$36 \mathrm{KD}$ マ

$45 \mathrm{KD}$ >

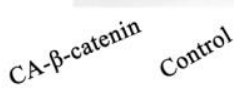

B

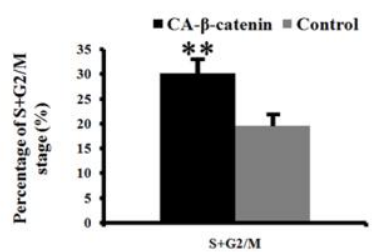

D
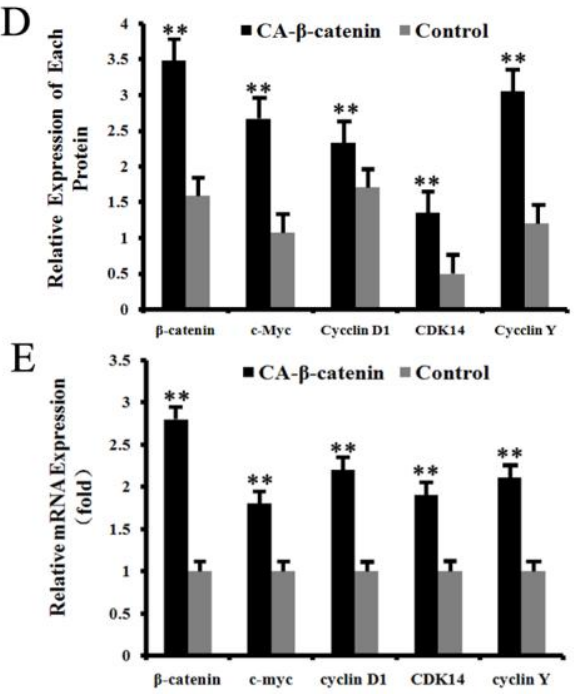

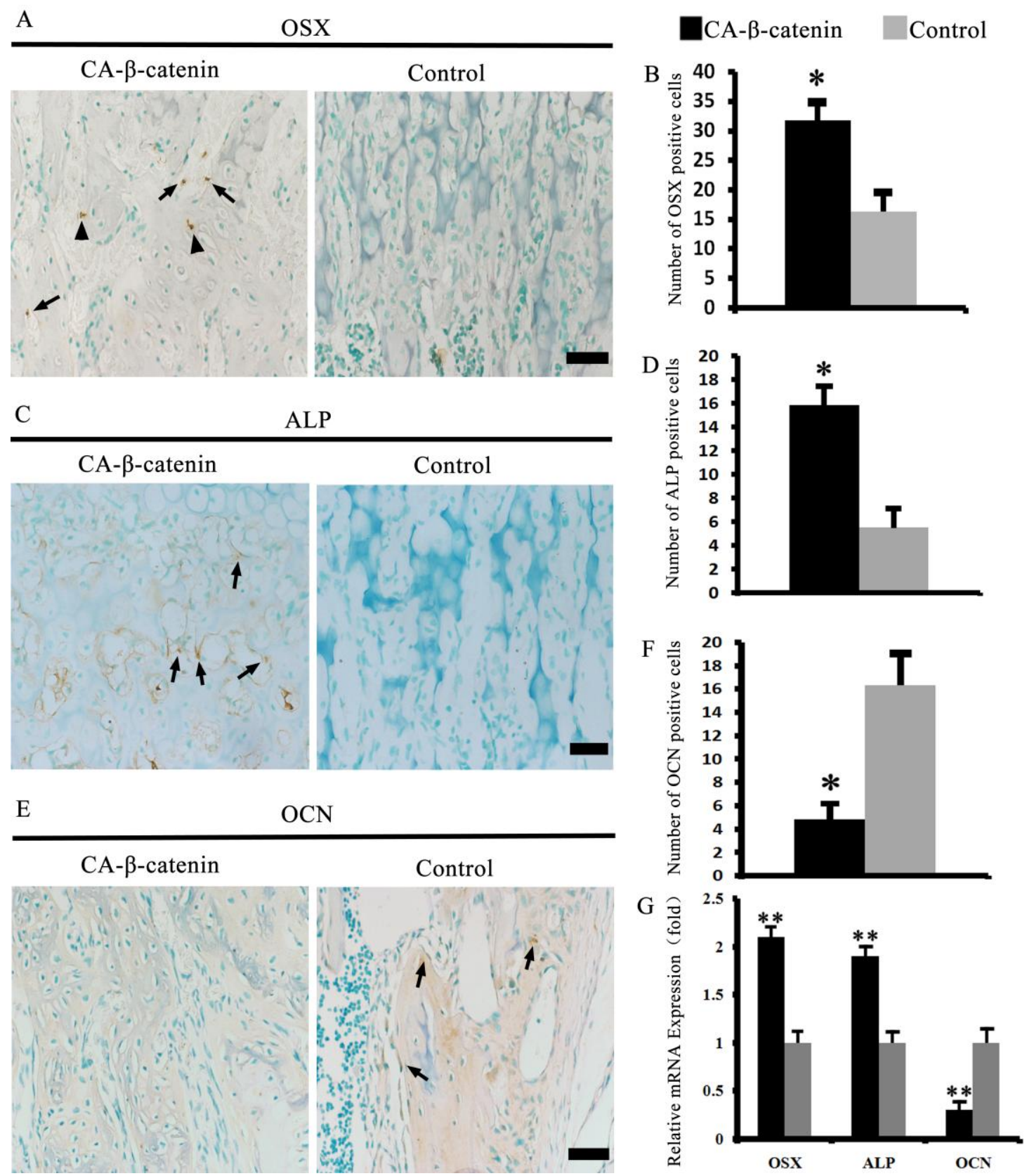